\title{
Morphometric Analysis of Thyroid Follicular Cells with Atypia of Undetermined Significance
}

Youngjin Kang · Yoo Jin Lee Jiyoon Jung · Youngseok Lee Nam Hee Won · Yang Seok Chae

Department of Pathology, Korea University Anam Hospital, Seoul, Korea

Received: January 15, 2016

Revised: April 2, 2016

Accepted: April 4, 2016

Corresponding Author

Yang Seok Chae, MD

Department of Pathology, Korea University Anam Hospital, 73 Inchon-ro, Seongbuk-gu, Seoul 02841, Korea

Tel: $+82-2-920-5595$

Fax: +82-2-920-6576

E-mail: chaeys21@korea.ac.kr

\begin{abstract}
Background: Atypia of undetermined significance (AUS) is a category that encompasses a heterogeneous group of thyroid aspiration cytology. It has been reclassified into two subgroups based on the cytomorphologic features: AUS with cytologic atypia and AUS with architectural atypia. The nuclear characteristics of AUS with cytologic atypia need to be clarified by comparing to those observed in Hashimoto thyroiditis and benign follicular lesions. Methods: We selected 84 cases of AUS with histologic follow-up, 24 cases of Hashimoto thyroiditis, and 26 cases of benign follicular lesions. We also subcategorized the AUS group according to the follow-up biopsy results into a papillary carcinoma group and a nodular hyperplasia group. The differences in morphometric parameters, including the nuclear areas and perimeters, were compared between these groups. Results: The AUS group had significantly smaller nuclear areas than the Hashimoto thyroiditis group, but the nuclear perimeters were not statistically different. The AUS group also had significantly smaller nuclear areas than the benign follicular lesion group; however, the AUS group had significantly longer nuclear perimeters. The nuclear areas in the papillary carcinoma group were significantly smaller than those in the nodular hyperplasia group; however, the nuclear perimeters were not statistically different. Conclusions: We found the AUS group to be a heterogeneous entity, including histologic follow-up diagnoses of papillary carcinoma and nodular hyperplasia. The AUS group showed significantly greater nuclear irregularities than the other two groups. Utilizing these features, nuclear morphometry could lead to improvements in the accuracy of the subjective diagnoses made with thyroid aspiration cytology.
\end{abstract}

Key Words: Atypia of undetermined significance; Morphometric analysis; Thyroid
Thyroid fine-needle aspiration (FNA) is widely used as an effective first-line screening test to differentiate thyroid lesions ${ }^{1-4}$ and the Bethesda System for Reporting Thyroid Cytopathology is used as a standard to interpret FNA specimens. "Atypia of undetermined significance (AUS)," known as Bethesda category III, is a category that encompasses a heterogeneous group of lesions containing follicular cells exhibiting architectural features and/ or nuclear atypia that exceed expected benign changes, but are not of sufficient magnitude to justify classification into any other categories. ${ }^{5}$

The AUS category has some limitations. First, it is more frequently diagnosed than previously recommended (threshold of $7 \%) .^{6-8}$ Second, it shows a considerable intra- and inter-observer variability. ${ }^{3,7-11}$ In addition, the risk of malignancy in the AUS category is not as low as previously thought; ${ }^{12-14}$ some researchers have considered the category as "waste garbage."

However, Shi et al. ${ }^{3}$ considered AUS to be an indispensable category in that it increases the sensitivity and decreases the false-positive and false-negative rates of thyroid FNA cytology. It is even helpful when the criteria are only partially fulfilled, especially for cases displaying subtle cytomorphological changes or scanty suspicious cells, cases obscured by blood or inflammation, or specimens compromised by air-drying artifacts.

AUS is a heterogeneous entity and includes nuclear and/or architectural changes that do not completely meet the qualitative or quantitative criteria to be suspicious for malignancy or follicular neoplasms. ${ }^{5}$ Based on the heterogeneity and subjective nature of this category, it has been suggested that this entity should be subcategorized into AUS with nuclear atypia and AUS with architectural atypia. ${ }^{15-21}$ Furthermore, AUS needs to be differentiated from benign lesions (category II), including Hashimoto thyroiditis and benign follicular lesions, as these differential diagnoses have meaningful clinical significance for patient follow-up.

Computerized nuclear morphometry is an objective, reproducible, and inexpensive tool to evaluate histological features. ${ }^{22,23}$ It has been suggested that nuclear morphometric parameters, such 
as nuclear perimeters and nuclear areas, may help differentiation between various thyroid lesions. ${ }^{24,25}$ Previous studies have revealed significant differences in nuclear areas and perimeters between benign and malignant lesions using thyroid aspiration cytology. ${ }^{26,27}$ However, the utilization of morphometric analysis in thyroid aspiration cytology is limited in clinical research as well as routine diagnoses. ${ }^{22}$

The aim of this study was to determine whether there is a significant difference in the nuclear morphometry findings between borderline and benign lesions found using thyroid aspiration cytology, in specimens diagnosed as AUS, Hashimoto thyroiditis, and benign follicular lesions.

\section{MATERIALS AND METHODS}

We selected 84 cases with thyroid liquid-based preparation cytologic slides originally diagnosed as "AUS with nuclear atypia" with histologic follow-up. For comparison, we also selected 24 cases of Hashimoto thyroiditis and 26 cases of benign follicular lesions (both Bethesda category II). All selected cases were from Korea University Anam Hospital, Seoul, Korea, from 2011 to 2013. All slides were submitted to digital image analysis using ImagePro 6 software (Media Cybernetics, Bethesda, MD, USA), and we measured the morphometric parameters, including the nuclear areas and perimeters (Fig. 1). Well-preserved, non-overlapping cells were usually selected, and tight three-dimensional clusters or papillae were excluded. The average number of measured cells per case was 19 (range, 11 to 32). The differences in morphometric parameters were separately compared between the three groups. Using SPSS ver. 14.0 software (SPSS Inc., Chicago, IL, USA), the mean nuclear perimeters and areas

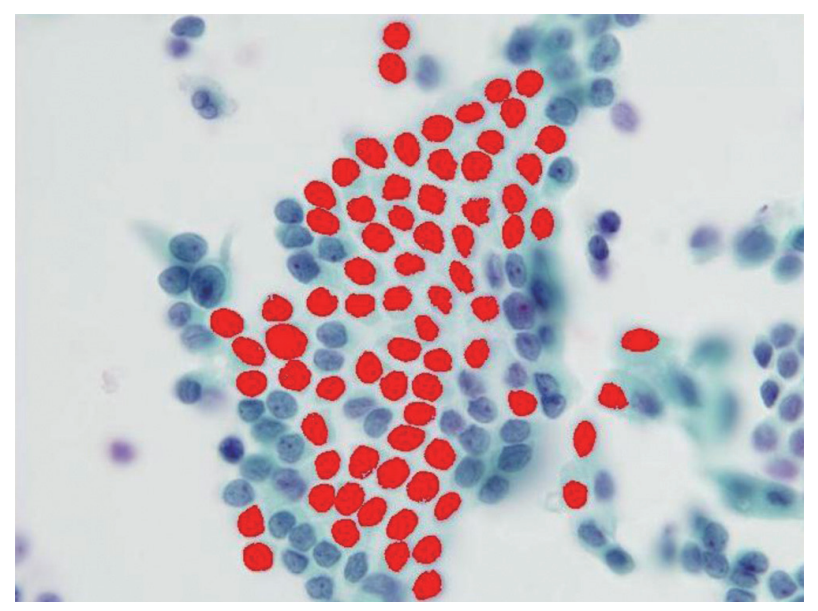

Fig. 1. Digital image analysis using ImagePro 6 software. The selected cells are marked in red. were compared via a Student's $\mathrm{t}$ test. This study was approved by the Institutional Review Board of Korea University Anam Hospital (AN15271-003).

\section{RESULTS}

The AUS group included 66 women and 18 men (mean age, 52 years), the Hashimoto thyroiditis group included 20 women and four men (mean age, 56 years), and the benign follicular lesion group included 24 women and two men (mean age, 55 years). There was no significant difference in the male:female ratio or in the mean age between groups (Table 1). The histologic diagnoses in the AUS group included conventional papillary thyroid carcinoma $(n=54)$, follicular variant of papillary thyroid carcinoma $(\mathrm{n}=10)$, nodular hyperplasia $(\mathrm{n}=18)$, and follicular neoplasm ( $\mathrm{n}=2)$ (Table 2, Fig. 2).

The mean nuclear area and perimeter were $19.360 \pm 4.881$ $\mu \mathrm{m}^{2}$ (mean \pm standard deviation) and $20.070 \pm 3.121 \mu \mathrm{m}$, $27.766 \pm 5.177 \mu \mathrm{m}^{2}$ and $21.112 \pm 2.693 \mu \mathrm{m}$, and $22.264 \pm$ $4.514 \mu^{2}$ and $18.206 \pm 2.036 \mu \mathrm{m}$ in the AUS group, Hashimoto thyroiditis group, and benign follicular lesion group, respectively (Table 3).

The nuclear areas in the AUS group were significantly smaller than those in the Hashimoto thyroiditis group $(\mathrm{p}<.001)$. The perimeters of the AUS group were not, however, significantly different from those of the Hashimoto thyroiditis group ( $\mathrm{p}=$ .140). The nuclear areas in the AUS group were also significantly smaller than those in the benign follicular lesion group ( $\mathrm{p}=$ .007), and the perimeters in the AUS group were also significantly longer than those in the benign follicular lesion group $(\mathrm{p}=$ .001) (Table 4).

As the AUS group showed heterogeneous follow-up biopsy

Table 1. Mean age and sex of the selected cases

\begin{tabular}{lccc}
\hline Variable & AUS & Hashimoto thyroiditis & Benign \\
\hline Mean age $(y r)$ & 52 & 56 & 55 \\
Sex & & & \\
$\quad$ Female & 66 & 20 & 24 \\
$\quad$ Male & 18 & 4 & 2 \\
\hline
\end{tabular}

AUS, atypia of undetermined significance.

Table 2. Follow-up pathologic diagnosis of cytologic slides

\begin{tabular}{lc}
\hline Pathologic diagnosis & No. \\
\hline PTC & 54 \\
PTC, follicular variant & 10 \\
Nodular hyperplasia & 18 \\
Follicular neoplasm & 2 \\
\hline
\end{tabular}

PTC, papillary thyroid carcinoma. 


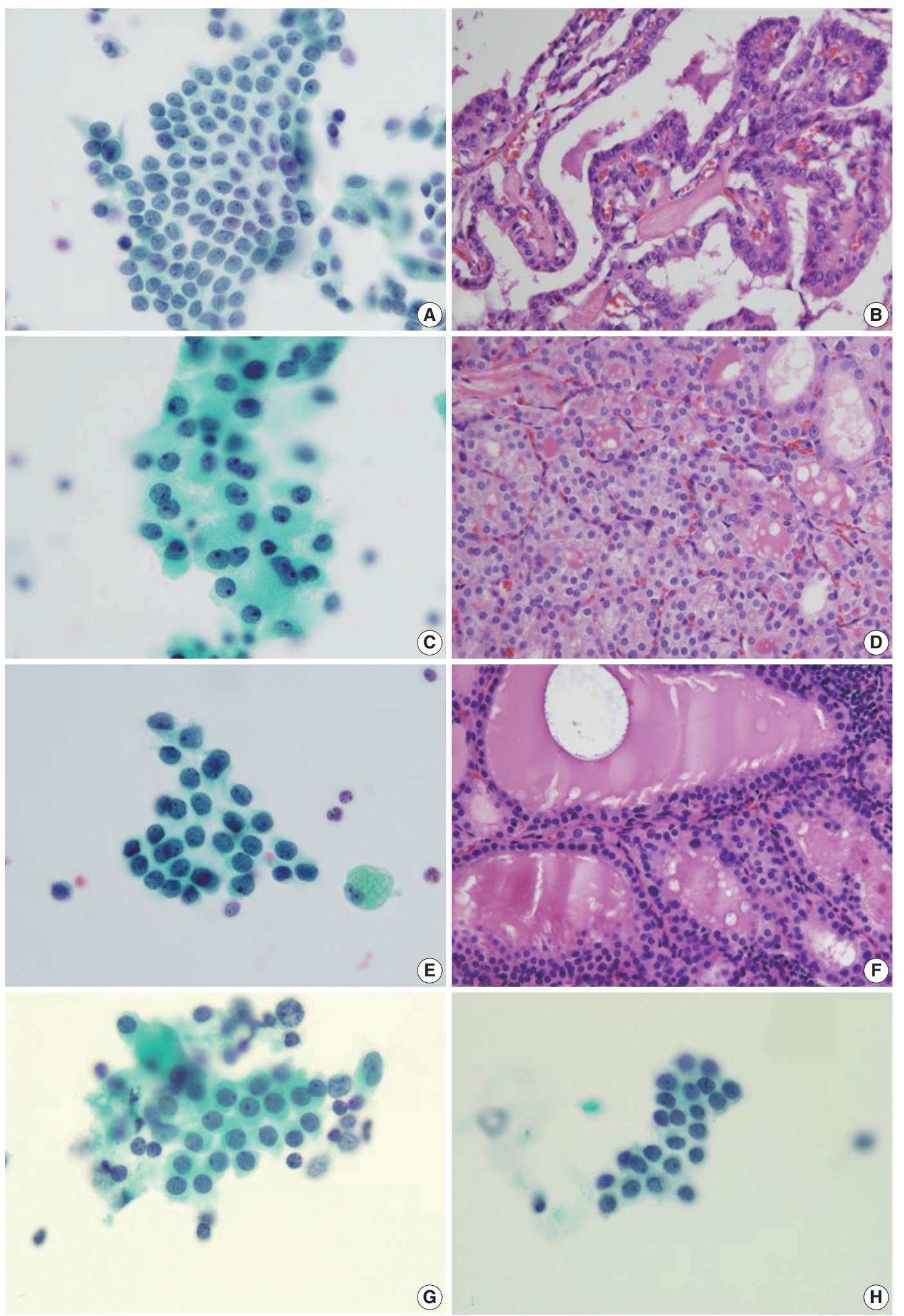

Fig. 2. (A, C, E) Cytologic slides for atypia of undetermined significance. Panels $B, D$, and $F$ are the corresponding histologic findings of panels $A, C$, and $E$, respectively. Finally, images are the cytologic slides for Hashimoto thyroiditis $(G)$ and benign follicular nodule $(H)$, respectively. They have no corresponding histologic slides, as a surgical resection was not performed. (B) Papillary carcinoma. (D) Papillary carcinoma (follicular variant). (F) Nodular hyperplasia. 
results, we subcategorized the results into a papillary carcinoma group (AUS finally diagnosed as papillary thyroid carcinoma) and a nodular hyperplasia group (AUS finally diagnosed as nodular hyperplasia). The papillary carcinoma group was composed

Table 3. Mean nuclear areas and perimeters of the three groups

\begin{tabular}{lccc}
\hline Variable & AUS & $\begin{array}{c}\text { Hashimoto } \\
\text { thyroiditis }\end{array}$ & Benign \\
\hline Area $\left(\mu \mathrm{m}^{2}\right)$ & $19.360 \pm 4.881$ & $27.766 \pm 5.177$ & $22.264 \pm 4.514$ \\
Perimeter $(\mu \mathrm{m})$ & $20.070 \pm 3.121$ & $21.112 \pm 2.693$ & $18.206 \pm 2.036$ \\
\hline
\end{tabular}

Values are presented as mean \pm standard deviation.

AUS, atypia of undetermined significance.

Table 4. Comparison of nuclear areas and perimeters between groups

\begin{tabular}{lcc}
\hline \multicolumn{1}{c}{ Group } & Two-tailed $\mathrm{p}$-value & Mean differences \\
\hline AUS vs. Hashimoto thyroiditis & & \\
$\quad$ Area $\left(\mu \mathrm{m}^{2}\right)$ & $<.001$ & -8.406 \\
$\quad$ Perimeter $(\mu \mathrm{m})$ & .140 & -1.042 \\
AUS vs. benign follicular lesions & & \\
$\quad$ Area $\left(\mu \mathrm{m}^{2}\right)$ & .007 & -2.904 \\
$\quad$ Perimeter $(\mu \mathrm{m})$ & .001 & 1.864 \\
Hashimoto thyroiditis vs. benign & & \\
follicular lesions & & \\
$\quad$ Area $\left(\mu \mathrm{m}^{2}\right)$ & $<.001$ & 5.502 \\
$\quad$ Perimeter $(\mu \mathrm{m})$ & $<.001$ & 2.906 \\
\hline
\end{tabular}

AUS, atypia of undetermined significance.

Table 5. Mean nuclear areas and perimeters in the AUS subgroups

\begin{tabular}{lcc}
\hline Variable & $\begin{array}{c}\text { AUS finally diagnosed } \\
\text { as PTC }\end{array}$ & $\begin{array}{c}\text { AUS finally diagnosed } \\
\text { as nodular hyperplasia }\end{array}$ \\
\hline Area $\left(\mu \mathrm{m}^{2}\right)$ & $18.711 \pm 4.283$ & $21.562 \pm 6.506$ \\
Perimeter $(\mu \mathrm{m})$ & $19.805 \pm 3.113$ & $21.147 \pm 3.235$ \\
\hline
\end{tabular}

Values are presented as mean \pm standard deviation.

AUS, atypia of undetermined significance; PTC, papillary thyroid carcinoma. of cases that were diagnosed as conventional papillary carcinoma and follicular variant papillary carcinoma. Follicular neoplasm was excluded from the comparison as there were only two cases.

The mean nuclear area and perimeter in the papillary carcinoma group was $18.711 \pm 4.283 \mu^{2}$ (mean \pm standard deviation) and $19.805 \pm 3.113 \mu \mathrm{m}$, respectively, and that in the nodular hyperplasia group was $21.562 \pm 6.506 \mu^{2}$ and $21.147 \pm 3.235$ $\mu \mathrm{m}$, respectively (Table 5).

The nuclear areas in the papillary carcinoma group were significantly smaller than those in the nodular hyperplasia group ( $\mathrm{p}$ $=.030)$, but the perimeters were not statistically different $(\mathrm{p}=$ .094). The nuclear areas in the papillary carcinoma group were also significantly smaller than those in the Hashimoto thyroiditis group ( $\mathrm{p}<.001)$, but the perimeters were not statistically different $(\mathrm{p}=.073)$. Similarly, the nuclear areas in the nodular hyperplasia group were significantly smaller than those in the Hashimoto thyroiditis group $(\mathrm{p}=.001)$, but the perimeters were not statistically different $(\mathrm{p}=.970)$. The perimeters in the nodular hyperplasia group were significantly longer than those in the benign follicular lesion group ( $\mathrm{p}=.001)$, but the nuclear areas were not statistically different $(\mathrm{p}=.674)$. Finally, the nuclear areas and perimeters in the papillary carcinoma group were significantly smaller and longer, respectively, than those in the benign follicular lesion group (Table 6).

\section{DISCUSSION}

FNA cytology has been widely used in the assessment of thyroid lesions. The Bethesda System for Reporting Thyroid Cytopathology has standardized the reporting of FNA cytology

Table 6. Comparison of nuclear areas and perimeters between subgroups

\begin{tabular}{lcc}
\hline Subgroup & Two-tailed $p$-value & Mean difference \\
\hline AUS finally diagnosed as PTC vs. AUS finally diagnosed as nodular hyperplasia & & -2.851 \\
$\quad$ Area $\left(\mu m^{2}\right)$ & .030 & -1.342 \\
$\quad$ Perimeter $(\mu \mathrm{m})$ & .094 & -0.702 \\
AUS finally diagnosed as nodular hyperplasia vs. benign follicular lesions & .674 & 2.941 \\
$\quad$ Area $\left(\mu m^{2}\right)$ & .001 & -6.204 \\
$\quad$ Perimeter $(\mu \mathrm{m})$ & & 0.034 \\
AUS finally diagnosed as nodular hyperplasia vs. Hashimoto thyroiditis & .001 & .970 \\
$\quad$ Area $\left(\mu m^{2}\right)$ & & -9.055 \\
$\quad$ Perimeter $(\mu \mathrm{m})$ & $<.001$ & -1.308 \\
AUS finally diagnosed as PTC vs. Hashimoto thyroiditis & .073 & -3.552 \\
$\quad$ Area $\left(\mu m^{2}\right)$ & & 1.599 \\
$\quad$ Perimeter $(\mu \mathrm{m})$ & .001 & .018 \\
AUS finally diagnosed as PTC vs. Benign follicular lesions & \\
$\quad$ Area $\left(\mu \mathrm{m}^{2}\right)$ & & \\
$\quad$ Perimeter $(\mu \mathrm{m})$ & & \\
\hline
\end{tabular}

AUS, atypia of undetermined significance; PTC, papillary thyroid carcinoma. 
findings for thyroid specimens. The diagnostic categories include non-diagnostic or unsatisfactory (category I), benign (category II), AUS (category III), follicular neoplasm or suspicious for a follicular neoplasm (category IV), suspicious for malignancy (category V), and malignant (category VI). The benign category (category II) has several subcategories, including benign follicular nodules and Hashimoto thyroiditis. The criteria for AUS, however, include lesions that do not fulfill the criteria for follicular neoplasms and papillary carcinomas, predominant Hurthle cells, and sample artifacts. The AUS category thus needs to be distinguished from both benign follicular nodules and Hashimoto thyroiditis.

The AUS lesion is a heterogeneous entity, and different institutions categorize it into multiple subgroups. These subgroups include AUS with cytologic atypia and AUS with architectural atypia. Among these subgroups, AUS with cytologic atypia is thought to be associated with an increased risk of malignancy. Mathur $e t ~ a l .{ }^{28}$ subcategorized 463 cases of thyroid aspiration cytology, re-reviewed as AUS, and the subgroup with nuclear atypia was found to have a greater risk of malignancy than any other subgroup (68\%), which was even greater than the overall risk of malignancy (39\%). In our study, 64 out of 84 cases of AUS (76\%) were found to be papillary carcinoma. Thus, the atypical nuclear features found in AUS should be evaluated thoroughly and differentiated from benign lesions, as it is critical for patient follow-up procedures.

Nevertheless, AUS is an essential, clinically significant category. Shi et al. ${ }^{3}$ have shown that eliminating the AUS category resulted in a consistent and considerable decrease of sensitivity in detecting thyroid lesions. The sensitivity of detecting papillary carcinoma was reduced from $100 \%$ to $27 \%$ when the AUS category was eliminated. Studies have also shown that eliminating the AUS category increases the false-negative and false-positive rates. $^{3}$ Without the AUS category, up to $53 \%$ of neoplastic thyroid lesions and $37 \%$ of papillary carcinomas would be underestimated as benign, and might not be clinically re-evaluated for months or years. Lastly, up to $38 \%$ of pathologically diagnosed benign lesions would be overestimated as a follicular neoplasm or suspicious for follicular neoplasm. These findings reveal that it is important to maintain the AUS category.

In these circumstances, additional objective morphological analysis is helpful in differentiating between AUS and benign lesions. Computerized nuclear morphometry is one of the solutions, which has the advantage of being both reproducible and inexpensive. ${ }^{22,23}$ Using nuclear morphometry, a number of parameters, such as nuclear size and shape, can be easily quantified.
The evaluation of these parameters has been documented to potentialize the diagnosis and the management of various neoplasms, including urinary bladder carcinoma, ${ }^{29}$ skin lymphoma, ${ }^{30}$ breast carcinoma, ${ }^{31}$ and soft tissue sarcoma. ${ }^{32}$ It has been suggested that nuclear morphometric parameters, such as nuclear areas and perimeters, may facilitate the differentiation between thyroid lesions. ${ }^{24,25}$ To date, however, the use of morphometric analysis in thyroid pathology has been limited.

Quantitative studies in pathology have enabled improvements in the accuracy of subjective diagnoses made in routine practice. ${ }^{26}$ Objective information gained through the quantification of nuclear morphological features may be useful in classifying different lesions. In thyroid follicular neoplasms, the most helpful parameters in the differential diagnosis are the nuclear parameters, including the mean nuclear area, the mean nuclear perimeter, the ratio of the largest to the smallest diameters of the nuclei, the coefficient of variation of the nuclear area, and the circular

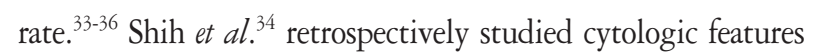
using computerized morphometry and clinical data in 118 cases. Multivariate logistic analysis showed that the parameters significantly related to recurrence were the nucleus-to-cell ratios, variations of the nuclear area, tumor sizes, and patient age. Aiad et $a l^{22}$ retrospectively studied 48 cases of different thyroid lesions to compare their parameters, including nuclear size, shape, perimeter, and area. Most parameters related to the sizes and the shapes of the nuclei were significantly higher in follicular variant papillary carcinoma than in follicular neoplasm. Also, nuclear areas and sizes were found to be the most reliable parameters to differentiate between follicular variant papillary carcinoma and follicular adenoma. Finally, Wright et al. ${ }^{27}$ evaluated 119 cases of FNA cytology of thyroid nodules and found significant differences in the nuclear areas and perimeters between the cases of multinodular goiters and follicular and papillary neoplasms, as well as between follicular adenomas and follicular and papillary carcinomas. These findings suggest that nuclear morphometry is useful in differentiating malignant from benign lesions.

We compared morphometric parameters, including the nuclear areas and perimeters, among groups categorized with AUS, Hashimoto thyroiditis, or benign follicular lesions. The AUS lesions showed significantly smaller nuclear areas with longer perimeters, suggesting that marked nuclear irregularity is statistically present. Most of the lesions were diagnosed as papillary carcinoma on the final histologic evaluation. However, the Bethesda System for Reporting Thyroid Cytopathology diagnostic criteria for papillary carcinoma includes enlarged nuclei. 
Previous studies using conventional smear slides revealed nuclear enlargement in papillary carcinoma. Murata et al. ${ }^{37}$ analyzed 39 cases of Pap-stained aspiration cytology smear specimens including nine cases of papillary carcinoma and revealed that papillary carcinomas had larger and more irregularly shaped nuclei than those of the benign groups. All selected cases in this study were liquid-based preparation cytologic slides, which may be a major cause of the smaller nuclei. Due to the inherent technical differences including alcohol fixation and the elimination of airdrying artifact cells, the nuclei may appear smaller in the liquidbased preparation specimens compared to the conventional smear specimens.

The nuclear irregularity of AUS lesions may also be a useful screening index in the routine diagnosis. In this study, the mean ratio of nuclear perimeter to nuclear area was 1.0367 in the AUS group, which was greater than the other two groups $(0.76035$ and 0.81773 in the Hashimoto thyroiditis group and the benign follicular group, respectively). A proper cut-off value would make the diagnosis of AUS much more objective, and thus further studies are needed to determine this proper cut-off value.

The AUS group is a heterogeneous entity, including histologic follow-up diagnoses of papillary carcinoma and nodular hyperplasia, but it is an essential category in spite of its limitations. We found that the AUS with cytologic atypia group was associated with an increased risk of malignancy, particularly due to its smaller nuclear areas and longer perimeters, indicating nuclear irregularity. Utilizing these features, nuclear morphometry would lead to improvements in the accuracy of the subjective diagnoses made using thyroid aspiration cytology. By determining a proper cut-off value, the diagnosis of AUS would be even more objective.

\section{Conflicts of Interest}

No potential conflict of interest relevant to this article was reported.

\section{REFERENCES}

1. DeMay RM. Cytopathology of false negatives preceding cervical carcinoma. Am J Obstet Gynecol 1996; 175(4 pt 2): 1110-3.

2. Greenblatt DY, Woltman T, Harter J, Starling J, Mack E, Chen H. Fine-needle aspiration optimizes surgical management in patients with thyroid cancer. Ann Surg Oncol 2006; 13: 859-63.

3. Shi Y, Ding X, Klein M, et al. Thyroid fine-needle aspiration with atypia of undetermined significance: a necessary or optional cate- gory? Cancer 2009; 117: 298-304.

4. Yang J, Schnadig V, Logrono R, Wasserman PG. Fine-needle aspiration of thyroid nodules: a study of 4703 patients with histologic and clinical correlations. Cancer 2007; 111: 306-15.

5. Cibas ES, Ali SZ; NCI Thyroid FNA State of the Science Conference. The Bethesda System For Reporting Thyroid Cytopathology. Am J Clin Pathol 2009; 132: 658-65.

6. Broome JT, Solorzano CC. The impact of atypia/follicular lesion of undetermined significance on the rate of malignancy in thyroid fine-needle aspiration: evaluation of the Bethesda System for Reporting Thyroid Cytopathology. Surgery 2011; 150: 1234-41.

7. Kholová I, Ludvíková M. Thyroid atypia of undetermined significance or follicular lesion of undetermined significance: an indispensable Bethesda 2010 diagnostic category or waste garbage? Acta Cytol 2014; 58: 319-29.

8. Ohori NP, Nikiforova MN, Schoedel KE, et al. Contribution of molecular testing to thyroid fine-needle aspiration cytology of "follicular lesion of undetermined significance/atypia of undetermined significance". Cancer Cytopathol 2010; 118: 17-23.

9. Bhasin TS, Mannan R, Manjari M, et al. Reproducibility of 'The Bethesda System for reporting Thyroid Cytopathology': a multicenter study with review of the literature. J Clin Diagn Res 2013; 7: 1051-4.

10. Cochand-Priollet B, Schmitt FC, Totsch M, Vielh P; European Federation of Cytology Societies Scientific Committee. The Bethesda terminology for reporting thyroid cytopathology: from theory to practice in Europe. Acta Cytol 2011; 55: 507-11.

11. Walts AE, Bose S, Fan X, et al. A simplified Bethesda System for reporting thyroid cytopathology using only four categories improves intra- and inter-observer diagnostic agreement and provides nonoverlapping estimates of malignancy risks. Diagn Cytopathol 2012; 40 Suppl 1: E62-8.

12. Chen JC, Pace SC, Khiyami A, McHenry CR. Should atypia of undetermined significance be subclassified to better estimate risk of thyroid cancer? Am J Surg 2014; 207: 331-6.

13. Ho AS, Sarti EE, Jain KS, et al. Malignancy rate in thyroid nodules classified as Bethesda category III (AUS/FLUS). Thyroid 2014; 24: 832-9.

14. Park VY, Kim EK, Kwak JY, Yoon JH, Moon HJ. Malignancy risk and characteristics of thyroid nodules with two consecutive results of atypia of undetermined significance or follicular lesion of undetermined significance on cytology. Eur Radiol 2015; 25: 2601-7.

15. Horne MJ, Chhieng DC, Theoharis C, et al. Thyroid follicular lesion of undetermined significance: evaluation of the risk of malignancy using the two-tier sub-classification. Diagn Cytopathol 2012; 40: 410-5. 
16. Nishino M, Wang HH. Should the thyroid AUS/FLUS category be further stratified by malignancy risk? Cancer Cytopathol 2014; 122: 481-3.

17. Olson MT, Clark DP, Erozan YS, Ali SZ. Spectrum of risk of malignancy in subcategories of 'atypia of undetermined significance'. Acta Cytol 2011; 55: 518-25.

18. Renshaw AA. Should "atypical follicular cells" in thyroid fine-needle aspirates be subclassified? Cancer Cytopathol 2010; 118: 186-9.

19. Renshaw AA. Subclassification of atypical cells of undetermined significance in direct smears of fine-needle aspirations of the thyroid: distinct patterns and associated risk of malignancy. Cancer Cytopathol 2011; 119: 322-7.

20. VanderLaan PA, Marqusee E, Krane JF. Usefulness of diagnostic qualifiers for thyroid fine-needle aspirations with atypia of undetermined significance. Am J Clin Pathol 2011; 136: 572-7.

21. Wu HH, Inman A, Cramer HM. Subclassification of "atypia of undetermined significance" in thyroid fine-needle aspirates. Diagn Cytopathol 2014; 42: 23-9.

22. Aiad H, Abdou A, Bashandy M, Said A, Ezz-Elarab S, Zahran A. Computerized nuclear morphometry in the diagnosis of thyroid lesions with predominant follicular pattern. Ecancermedicalscience 2009; 3: 146.

23. Hamilton PW, Allen DC. Morphometry in histopathology. J Pathol 1995; 175: 369-79.

24. Fadda G, Rabitti C, Minimo C, et al. Morphologic and planimetric diagnosis of follicular thyroid lesions on fine needle aspiration cytology. Anal Quant Cytol Histol 1995; 17: 247-56.

25. Słowińska-Klencka D, Klencki M, Sporny S, Lewiński A. Karyometric analysis in the cytologic diagnosis of thyroid lesions. Anal Quant Cytol Histol 1997; 19: 507-13.

26. Artacho-Pérula E, Roldán-Villalobos R, Blanco-García F, BlancoRodríguez A. Objective differential classification of thyroid lesions by nuclear quantitative assessment. Histol Histopathol 1997; 12: 425-31.

27. Wright RG, Castles H, Mortimer RH. Morphometric analysis of thyroid cell aspirates. J Clin Pathol 1987; 40: 443-5.

28. Mathur A, Najafian A, Schneider EB, Zeiger MA, Olson MT. Malignancy risk and reproducibility associated with atypia of undetermined significance on thyroid cytology. Surgery 2014; 156: 1471-6.

29. Kapur U, Antic T, Venkataraman G, et al. Validation of World Health Organization/International Society of Urologic Pathology 2004 classification schema for bladder urothelial carcinomas using quantitative nuclear morphometry: identification of predictive features using bootstrap method. Urology 2007; 70: 1028-33.

30. Lira M, Schenka AA, Magna LA, et al. Diagnostic value of combining immunostaining for CD3 and nuclear morphometry in mycosis fungoides. J Clin Pathol 2008; 61: 209-12.

31. Cui Y, Koop EA, van Diest PJ, Kandel RA, Rohan TE. Nuclear morphometric features in benign breast tissue and risk of subsequent breast cancer. Breast Cancer Res Treat 2007; 104: 103-7.

32. Kazanowska B, Jelen M, Reich A, Tarnawski W, Chybicka A. The role of nuclear morphometry in prediction of prognosis for rhabdomyosarcoma in children. Histopathology 2004; 45: 352-9.

33. Nagashima T, Suzuki M, Oshida M, et al. Morphometry in the cytologic evaluation of thyroid follicular lesions. Cancer 1998; 84: 115-8.

34. Shih SR, Chang YC, Li HY, et al. Preoperative prediction of papillary thyroid carcinoma prognosis with the assistance of computerized morphometry of cytology samples obtained by fine-needle aspiration: preliminary report. Head Neck 2013; 35: 28-34.

35. Tseleni-Balafouta S, Kavantzas N, Paraskevakou H, Davaris P. Computerized morphometric study on fine needle aspirates of cellular follicular lesions of the thyroid. Anal Quant Cytol Histol 2000; 22: 323-6.

36. Wang SL, Wu MT, Yang SF, Chan HM, Chai CY. Computerized nuclear morphometry in thyroid follicular neoplasms. Pathol Int 2005; 55: 703-6.

37. Murata S, Mochizuki K, Nakazawa T, et al. Morphological abstraction of thyroid tumor cell nuclei using morphometry with factor analysis. Microsc Res Tech 2003; 61: 457-62. 